\title{
Molecular characterization of circulating tumor cells in pancreatic ductal adenocarcinoma: potential diagnostic and prognostic significance in clinical practice
}

\author{
Xudong Zhao ${ }^{1 \#}$, Yongsu Ma ${ }^{1 \#}$, Xiu Dong ${ }^{2 \#}$, Zhengkui Zhang ${ }^{1}$, Xiaodong Tian $^{1}$, Xiaohang Zhao ${ }^{2}$, \\ Yinmo Yang ${ }^{1}$
}

${ }^{1}$ Department of General Surgery, Peking University First Hospital, Beijing, China; ${ }^{2}$ State Key Laboratory of Molecular Oncology, National Cancer Center/Cancer Hospital, Chinese Academy of Medical Sciences \& Peking Union Medical College, Beijing, China

Contributions: (I) Conception and design: X Zhao, Y Ma, X Zhao, Y Yang; (II) Administrative support: X Tian, X Zhao, Y Yang; (III) Provision of study material or patients: Z Zhang, X Tian, X Zhao, Y Yang; (IV) Collection and assembly of data: X Zhao, Y Ma, X Dong; (V) Data analysis and interpretation: X Zhao, Y Ma, X Dong; (VI) Manuscript writing: All authors; (VII) Final approval of manuscript: All authors.

\#These authors contributed equally to this work.

Correspondence to: Yinmo Yang, MD, PhD, FACS. Professor and Chair, Department of General Surgery, Peking University First Hospital, 8 Xishiku Street, Xicheng District, Beijing 100034, China. Email: yangyinmo@263.net; Xiaohang Zhao, PhD. Professor, State Key Laboratory of Molecular Oncology, National Cancer Center/Cancer Hospital, Chinese Academy of Medical Sciences, 17 Panjiayuan Nanli, Chaoyang District, Beijing 100021, China. Email: zhaoxh@cicams.ac.cn.

Background: The clinical value of heterogeneous sub-populations of circulating tumor cells (CTCs) in pancreatic ductal adenocarcinoma (PDAC) remains unclear.

Methods: Peripheral blood samples were obtained from 67 PDAC patients. CTCs were isolated by employing CD45 negative enrichment technique and further characterized for epithelial to mesenchymal transition (EMT) or human equilibrative nucleoside transporter-1 (hENT-1). The relationships between CTCs sub-phenotypes with clinicopathological factors or post-operative recurrence in PDAC patients were analyzed.

Results: EMT related CTCs could be isolated and identified from the $81 \%$ of patients (54/67), and both the total count (median: 5 vs. 17/mL, $\mathrm{P}<0.0001$ ) and M-CTC percentage (median: 0.2 vs. $0.345, \mathrm{P}=0.0244$ ) of CTCs could differentiate local/regional with metastatic disease. Multivariate analysis showed that both AJCC stage $(\mathrm{P}=0.025)$ and $\mathrm{M}-\mathrm{CTC}$ percentage $(\mathrm{P}=0.001)$ were independent prognostic indicators of recurrence free survival (RFS) in resected patients. Moreover, Kaplan-Meier survival analysis showed that M-CTC after 2 courses of chemotherapy was significantly associated with inferior RFS (49.5 weeks vs. undefined, $\mathrm{P}=0.0288$ ). No significant correlation in hENT-1 expression was found between CTCs and matched tumor tissues, and further multivariate analysis suggested hENT-1 expression in CTCs as independent prognostic factor for RFS (P=0.016). Patients with low hENT-1 expression in CTCs had decreased RFS (32 weeks vs. undefined, $\mathrm{P}=0.0337)$.

Conclusions: CTCs could be the promising diagnostic biomarkers in PDAC patients, and phenotypic profiling of CTCs based on EMT or hENT-1 could help establish novel prognostic biomarkers in resected patients undergoing adjuvant gemcitabine-based chemotherapy.

Keywords: Circulating tumor cells (CTCs); Pancreatic ductal adenocarcinoma (PDAC); Epithelial to mesenchymal transition (EMT); human equilibrative nucleoside transporter-1 (hENT-1)

Submitted Mar 19, 2020. Accepted for publication May 07, 2020.

doi: $10.21037 / \mathrm{hbsn}-20-383$

View this article at: https://dx.doi.org/10.21037/hbsn-20-383 


\section{Introduction}

Pancreatic ductal adenocarcinoma (PDAC) is estimated to become the second leading cause of cancer associated death in the United States by 2030 (1). Due to the lack of appropriate screening and early detection methods, the majority of PDAC patients are diagnosed as advanced or metastatic stage (2). It is estimated that only $10-20 \%$ patients are suitable for tumor resection; however, the recurrence rate is as high as $65.6-76.7 \%(2,3)$. Such a high rate of recurrence should be attributed to the failure of systemic therapy in eradicating the post-operative residual micrometastatic disease (4). Nowadays, adjuvant therapy with either gemcitabine or 5 -fluorouracil (5-FU) based combination regimens including FOLFIRINOX has been considered as the standard treatment for PDAC, however, no established biomarkers currently could be used to select the more appropriate treatment strategy for the individual patient to improve the prognosis $(5,6)$.

A promising biomarker is the human equilibrative nucleoside transporter-1 (hENT-1), which mediates the entry of nucleoside analogs into cells, such as gemcitabine $(7,8)$. Several clinical studies have suggested that high expression of hENT-1 was associated with increased disease-free survival (DFS) and overall survival (OS) among resected PDAC patients undergoing adjuvant gemcitabinebased treatment $(9,10)$. Nevertheless, other controversial results showed that patient stratification based on hENT-1 expression was insufficient to be adopted into current clinical decision-making processes $(11,12)$. It is still not fully understood how hENT-1 expression in resected tumor tissues may be correlated to the chemosensitivity of the residual occult micrometastases (13). Therefore, reliable biomarkers with high sensitivity and specificity for early diagnosis, risk stratification and prognostic estimation of PDAC are urgently needed $(14,15)$.

Circulating tumor cells (CTCs) are disseminated neoplastic cells released from the primary tumor or metastatic foci into the circulatory system (16-18). CTCs enumeration in the peripheral blood has the potential in diagnosis and prognosis prediction in PDAC patients $(19,20)$. Furthermore, epithelial to mesenchymal transition (EMT) plays an important role in the dissemination of such cancer cells (21). During EMT, CTCs lose some of their epithelial properties to gain characteristics of mesenchymal phenotype, resulting in increased mobility and invasion ability to facilitate metastasis (21-23). However, the actual distribution characteristics of EMT related CTCs in blood and their potential clinical significance in PDAC patients are still not fully elaborated. Moreover, as metastasis-driven precursors, CTCs should have the potential to assess individual micrometastases burden $(24,25)$. Therefore, whether hENT1 expression in CTCs could serve as an alternative prognostic biomarker in patients undergoing adjuvant gemcitabine-based chemotherapy is of great interest $(26,27)$.

In this study, we aimed to analyze the correlation of CTCs sub-phenotypes with clinicopathological factors and post-operative recurrence of PDAC patients. In particular, we differentiated CTCs into three distinct sub-phenotypes (E-CTC, E/M-CTC and M-CTC) and performed semiquantitative analysis of hENT-1 expression in CTCs to determine its potential for patient stratification for recurrence free survival (RFS). To our knowledge, this is the first comprehensive study to explore the utility of heterogeneous sub-populations of CTCs in the diagnosis and prognosis of PDAC. We present the following article in accordance with the REMARK reporting checklist (available at https://hbsn.amegroups.com/article/view/10.21037/ hbsn-20-383/rc).

\section{Methods}

\section{Subjects}

This study enrolled 67 consecutive PDAC patients who visited Peking University First Hospital from March 2018 to April 2019. In addition, 10 healthy donors and 6 chronic pancreatitis patients were enrolled as controls (Figure 1A). The study was conducted in accordance with the Declaration of Helsinki (as revised in 2013). The study was approved by the Institutional Review Board at the Peking University First Hospital (No. 2018 keyan-15) and informed consent was taken from all individual participants.

Patients or healthy individuals met the following criteria: had no medical history of malignancy, and were treatmentnaïve before blood collection. For 45 resected patients, diagnosis was confirmed by pathology, while diagnosis of 22 unresectable PDAC was supported by biopsy of either the primary tumor or metastases. All participants had their first blood sample drawn at the time of diagnosis. In a subset of 18 resected patients, blood sample collection was also performed at the time points of pre-chemotherapy and after 2 cycles of gemcitabine-based adjuvant chemotherapy.

The patient demographics included the age, gender, tumor location, CA19-9 level, TNM stage and survival outcome. Among patients who underwent surgical resection, further pathological information such as tumor size, differentiation grade, lymph nodes status, perineural 
A

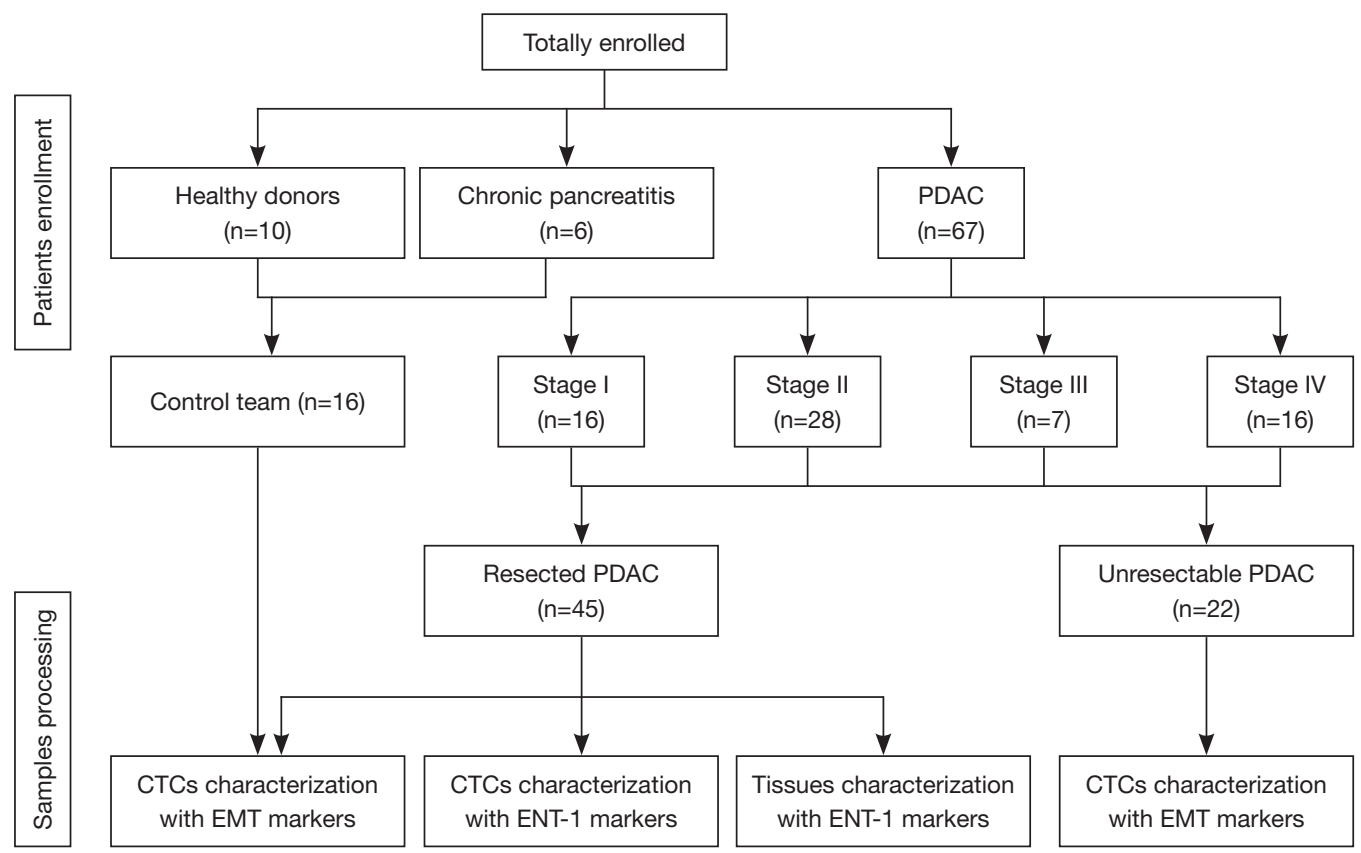

B

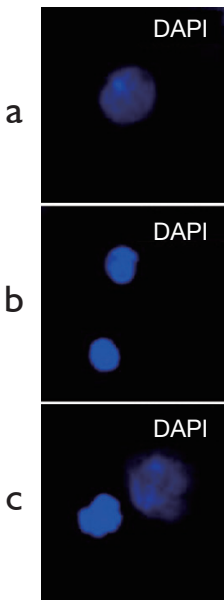

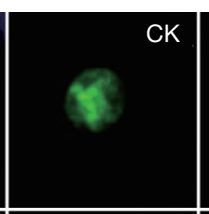

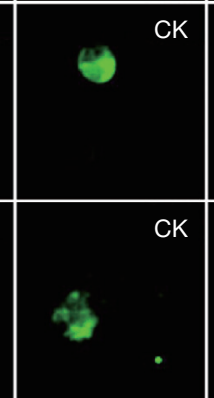

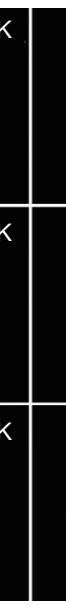
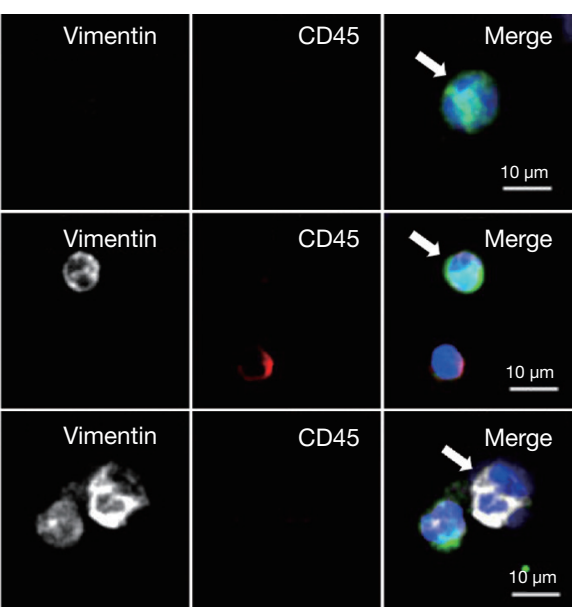

C

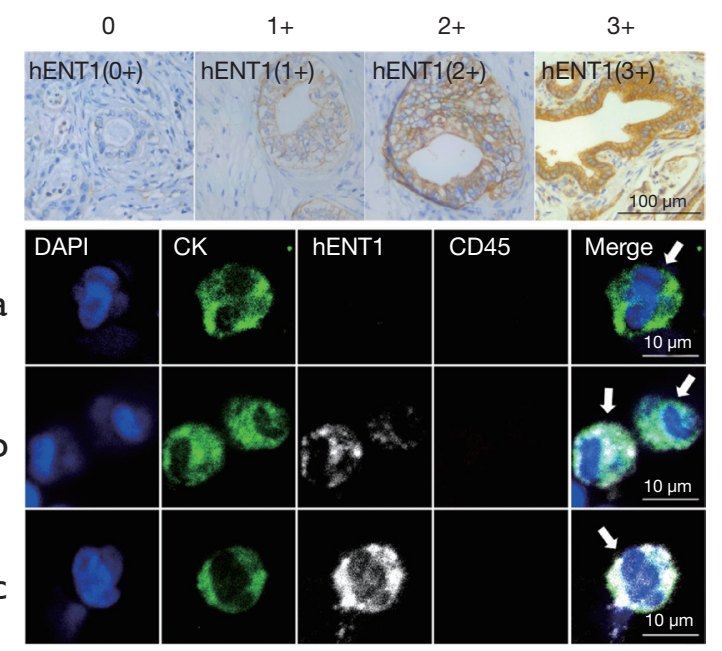

Figure 1 Study cohort flowchart and CTCs immunofluorescence analysis. (A) Study cohort flowchart for patients enrollment and samples processing. (B) Representative images for CTCs subtypes characterized with EMT related markers. (a) Top row: epithelial CTCs (E-CTC) with $\mathrm{CK}^{+} /$vimentin $/ \mathrm{CD}^{-} 5^{-} / \mathrm{DAPI}^{-}$(white arrow); (b) middle row: epithelial/mesenchymal hybrid CTCs (E/M-CTC) with $\mathrm{CK}^{-} / \mathrm{vimentin}^{+} /$ $\mathrm{CD}^{-} 5^{-} / \mathrm{DAPI}^{+}$(white arrow); (c) bottom row: mesenchymal CTCs (M-CTC) with $\mathrm{CK}^{-} /$vimentin ${ }^{+} / \mathrm{CD}^{-} 5^{-} / \mathrm{DAPI}^{+}$(white arrow). (C) Differential expression levels of hENT-1 in CTCs and tumor tissues in PDAC patients. CTCs with different hENT-1 staining intensity were shown (white arrow): no staining (a), weakly positive and moderately positive (b, right and left), strongly positive (c). 
and microvascular invasion state were collected.

Based on patient preference, 45 resected patients received gemcitabine-based adjuvant therapy and were followed routinely every 3-6 months to monitor tumor recurrence. Follow-up was terminated on October 30, 2019. Tumor recurrence was defined based on the presence of imaging recognizable lesions. RFS was determined as the interval between resection and the diagnosis of tumor recurrence or the end of follow-up. In particular, the treatment plan was independent of CTCs status in this observational prospective study.

\section{CTCs isolation}

Blood samples were processed and isolated via CD45 negative enrichment method. Firstly, peripheral blood mononuclear cells (PBMCs) were collected by density gradient centrifugation using Histopaque-1077 solution (Sigma-Aldrich). Secondly, the PBMCs were mixed with microbeads (Miltenyi Biotec) conjugated to monoclonal anti-human CD45 antibodies. CD $45^{+}$white blood cells were depleted by flowing through a LS Column (Miltenyi Biotec) on a magnetic separation (Miltenyi Biotec). Then, unlabeled $\mathrm{CD}^{4} 5^{-}$cells were spotted on glass slides for immunofluorescence staining.

\section{Immunofluorescence analysis}

Cells were washed three times with PBS, fixed in 2\% PFA for $40 \mathrm{~min}$, and then penetrated with $0.2 \%$ triton $/ 2 \%$ BSA for $45 \mathrm{~min}$. Then, the cells were incubated with anti-CKFITC (Miltenyi Biotec), anti-vimentin-alexa fluor 647 (Cell Signaling), anti-CD45-PE (Miltenyi Biotec) for $1 \mathrm{~h}$ at room temperature. The slides were washed three times with PBS and stained with DAPI (Sigma-Aldrich) and ProLong Gold anti-fade mountant (Invitrogen). After staining, the slides were scanned by the Leica confocal microscope. CTCs were classified into three subtypes: (I) epithelial CTCs (E-CTC), $\mathrm{CK}^{+} /$vimentin ${ }^{-} / \mathrm{CD}^{2} 5^{-} / \mathrm{DAPI}^{+}$, (II) epithelial/ mesenchymal hybrid CTCs (E/M-CTC), $\mathrm{CK}^{+} /$vimentin $^{+} /$ $\mathrm{CD}^{-} / \mathrm{DAPI}^{+}$, (III) mesenchymal CTCs (M-CTC), $\mathrm{CK}^{-} /$ vimentin ${ }^{+} / \mathrm{CD}^{-} 5^{-} / \mathrm{DAPI}^{+}$(Figure $1 B$ ).

In addition, cells were incubated with anti-ENT-1 primary antibody (Abcam) and anti-rabbit-alexa fluor 647 conjugated secondary antibody (Cell Signaling) (hENT-1 antibody assessment provided in Appendix 1). The staining intensity for hENT-1 in individual CTC and the percentage of hENT-1 positive CTCs were scored based on the immunohistochemical scoring system $(28,29)$. The staining intensity of hENT-1 was divided into four levels: 0, no staining; 1 , weakly positive; 2 , moderately positive and 3 , strongly positive (Figure 1C); the positive percentage was assigned a score ranging from 0 to $3: 0, \leq 5 \%$ positive cells; $1,5-25 \%$ positive cells; $2,25-50 \%$ positive cells; $3,>50 \%$ positive cells. The final hENT-1 score for each patient was obtained by summing these two scores. According to the median score in our cohort, CTCs with hENT-1 score higher or lower than the median score were classified as high or low hENT-1 expression, respectively.

\section{KRAS gene mutation analysis}

Genomic DNA was isolated from CTCs and amplified using REPLI-g Single Cell Kit (QIAGEN) for analysis of KRAS gene mutation following the manufacturer's protocol. Meanwhile, genomic DNA was extracted from tumor tissues using GeneRead DNA FFPE kit (QIAGEN) following the manufacturer's protocol, and then used for KRAS gene mutation analysis by Sanger sequencing.

\section{Immunobistochemistry analysis}

Immunohistochemistry (IHC) analyses were performed as previously described $(28,29)$. Formalin-fixed, paraffinembedded (FFPE) tumor tissues from PDAC patients were deparaffinized with xylene, rehydrated with ethanol and boiled for 20 minutes in microwave for antigen retrieval in a $10-\mathrm{mM}$ citrate buffer $(\mathrm{pH} 6)$. Then, the samples were blocked by $3 \% \mathrm{H}_{2} \mathrm{O}_{2}$. After washing, the slides were incubated with anti-ENT-1 antibody, and evaluated by two experienced histopathologists who were blinded to treatment group and outcomes. The intensity of cytoplasmic and membrane staining in tumor cells was scored from 0 to 3 (Figure 1C). The positive percentage of neoplastic cells was scored as follows: 0 , no positive neoplastic cells; $1,<50 \%$ positive cells; $2,50-80 \%$ positive cells; $3, \geq 81 \%$ positive cells. The score for individual patient was calculated by summing the scores both in the intensity and the positive percentage of tumor cells stained. Tumors with score of 0-4 were classified as low hENT-1 expression and tumors with score of 4-6 as high hENT-1 expression.

\section{Statistical analysis}

All statistical analyses were performed using SPSS 
25.0 (IBM Corp., Armonk, NY, USA) and Prism 7.0 (Graphpad, La Jolla, CA, USA). Data were reported as count and percentages for categorical data, and mean \pm standard deviation (SD) with ranges for continuous data. Comparisons between two groups were analyzed using Chi-square test or Mann-Whitney U test. The KruskalWallis test was used for assessing the differences if the data were not normally distributed, and the Dunn's multiple comparison test was adopted for data comparison between any two groups. Patient characteristics associated with post-operative recurrence were identified by univariate and multivariate Cox-regression analyses. Significant variables in the univariate Cox-regression analysis $(\mathrm{P}<0.20)$ were selected for multivariate Cox-regression analysis with a backward stepwise selection (Wald). Kaplan-Meier curve with a log-rank test was performed for RFS analysis. Receiver operating characteristic curve (ROC) analysis with maximal Youden index values was applied to determine best cut-off values. Concordance between hENT-1 expression in CTCs and matched tumor tissues was determined by calculation of intra-class correlation coefficients. All statistical assessments were two-tailed, and $\mathrm{P}$ values $<0.05$ were considered statistically significant.

\section{Results}

KRAS gene mutation analysis of CTCs and matched tumor tissues in individual patients

KRAS is the most frequently mutated gene in PDAC, and mutated KRAS could be found in $70-95 \%$ of PDAC patients (30). In order to validate PDAC origin of CTCs isolated and identified in our study, we performed KRAS mutation analysis in 5 patients that had both CTCs and matched FFPE tumor tissue available. The results showed that KRAS mutation subtype of FFPE tumor tissue was consistent with that of CTCs in all 5 patients analyzed, indicating that the CTCs were disseminated from primary tumors (Figure 2A).

\section{The correlation of EMT related CTCs with the development and metastasis of PDAC}

EMT related CTCs were found in 54/67 (81\%) PDAC patients, with $53 / 67$ (79\%) as E-CTC, 51/67 (76\%) as E/ M-CTC, 47/67 (70\%) as M-CTC, respectively. In contrast, only $2 / 10$ healthy volunteers and $1 / 6$ chronic pancreatitis patients had E-CTC-like cells (median: 6 vs. $0 / \mathrm{mL}$, $\mathrm{P}<0.0001)$. Specially, the E-CTC-like cells in chronic pancreatitis patients were circulating epithelial cells (CECs), indicating that CTCs characterized with epithelial markers might have limited ability as a screening tool to identify PDAC patients (31). Therefore, CTCs could be a potential diagnostic biomarker for PDAC, especially M-CTC (CTCs total count: AUROC $=0.808 ;$ M-CTC count: AUROC $=0.851)$.

CTCs were found in $75 \%, 79 \%, 86 \%$, and $88 \%$ of AJCC stage I, II, III, and IV patients, respectively. Among these stage groups, median CTCs numbers were 4 [2-5], 5 [3-7], 10 [8-15], $16[13-20] / \mathrm{mL}$, respectively (Figure $2 B$ ). In order to test the ability of CTCs to differentiate patients with or without metastases, we found a significant difference in CTCs total count \{median: 5 [2-7] vs. 17 [14-20]/mL, $\mathrm{P}<0.0001\}$ and M-CTC percentage [median: $0.2(0-0.38)$ vs. 0.35 (0.27-0.43), $\mathrm{P}=0.0244$ ] between the patients with local/ regional and metastatic cancer. Meanwhile, no differences in the median proportion of E-CTC or E/M-CTC were found between two groups $(\mathrm{P}=0.583, \mathrm{P}=0.205$, respectively) (Figure 2C,2D).

Furthermore, ROC analysis showed that a value of $11 \mathrm{CTCs} / \mathrm{mL}$ blood or 0.235 in M-CTC percentage were the optimum cut-off for metastatic status in patients with PDAC, with the sensitivity of 0.929 and 0.857 , and the specificity of 0.887 and $0.566(\mathrm{P}<0.0001, \mathrm{P}=0.027$, respectively), and both of the values had better performance than CA19-9 level to distinguish metastases (Figure 2E).

\section{The correlation between CTCs enumeration or M-CTC percentage with clinicopathological features}

As shown in Table 1, the presence of CTCs was positively correlated with AJCC TNM stage $(\mathrm{P}<0.001)$ and distant metastasis $(\mathrm{P}<0.001)$, while patients with distant metastases had higher M-CTC percentage $(\mathrm{P}=0.025)$. The other clinicopathological features showed no significant relationship with CTCs total count or individual M-CTC ratio. Moreover, in patients who underwent curative resection $(n=45)$, clinicopathological parameters were not significantly correlated with CTCs total count or individual M-CTC percentage.

\section{The correlation of EMT related CTCs with the prognosis of PDAC}

The best cut-off value of CTCs total count and subgroup percentage for evaluating prognostic significance were explored by ROC analysis. A cut-off of $3 / \mathrm{mL}$ in CTCs total 
A Patient \#1 KRAS G12D (GGT ${ }^{\text {Pro }} \rightarrow$ GAT $\left.{ }^{\text {Leu }}\right)$

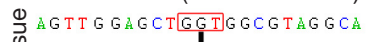

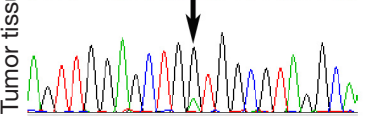

KRAS G12D $\left(\mathrm{GGT}^{\mathrm{Pro}} \rightarrow \mathrm{GAT}^{\mathrm{Leu}}\right)$
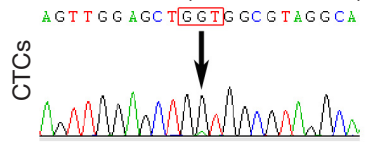

D

a

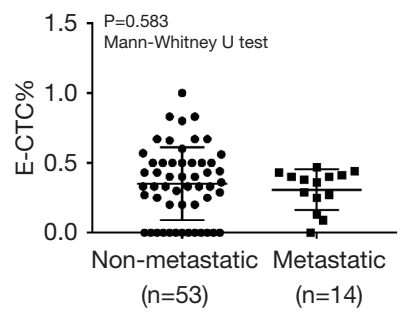

Patient \#2

KRAS G12D (GGT Pro

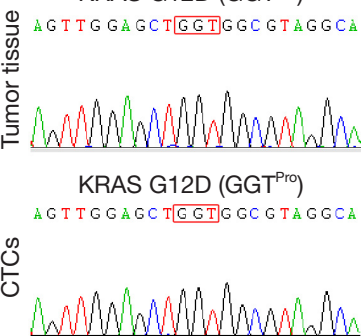

B

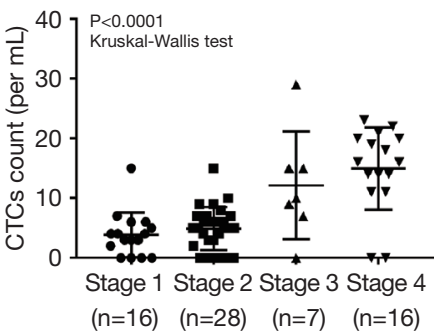

C

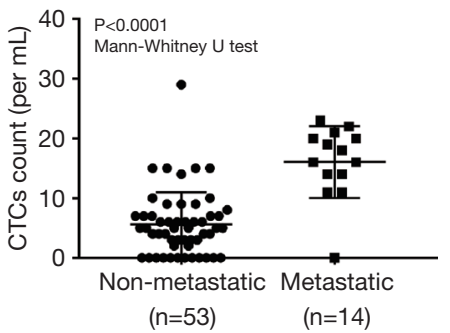

Figure 2 Evaluation of EMT related CTCs as diagnostic markers of PDAC. (A) KRAS gene mutation analysis. Sanger sequencing data for KRAS codon 12 mutations in CTCs and matched tumor tissues from 2 PDAC patients were presented. Patient \#1 had a G12D mutation and patient \#2 had no mutation. (B) CTCs enumeration correlated with AJCC stage in PDAC $(\mathrm{P}<0.0001)$. (C,D) CTCs total count $(\mathrm{P}<0.0001)$ and M-CTC ratio $(\mathrm{P}=0.024)$ in peripheral blood correlated with PDAC metastatic status. (E) ROC analysis of CTCs total count and M-CTC percentage in discriminating local/regional from metastatic disease. (CTCs count combined with M-CTC percentage: AUROC $=0.897$, 95\% CI: 0.774-1.000, P<0.0001; CTCs total count: AUROC =0.894, 95\% CI: 0.771-1.000, P<0.0001; M-CTC percentage: AUROC $=0.693$, 95\% CI: $0.553-0.832, \mathrm{P}=0.027)$.

count (AUROC $=0.660$, sensitivity $=85.7 \%$ and specificity $=37.5 \%$ ) and $23.5 \%$ in M-CTC percentage (AUROC $=0.759$, sensitivity $=71.4 \%$ and specificity $=75.0 \%$ ) at baseline demonstrated the maximal AUROC in predicting recurrence.

Univariate analysis of RFS in resected patients $(n=45)$ revealed that AJCC TNM stage $(\mathrm{P}=0.034)$, microvascular invasion $(\mathrm{P}=0.005)$, and $\mathrm{M}-\mathrm{CTC}$ percentage $(\mathrm{P}=0.001)$ in peripheral blood were prognostic indicators. Further multivariate analysis revealed that both AJCC stage $(\mathrm{P}=0.025)$ and $\mathrm{M}$-CTC percentage $(\mathrm{P}=0.001)$ were independent prognostic indicators for PDAC who underwent curative resection (Table 2).

Kaplan-Meier survival analysis showed that patients presented with advanced AJCC stage (33 vs. 58 weeks, $\mathrm{HR}=2.665$, 95\% CI: 1.123-6.325, log-rank test $\mathrm{P}=0.0267$ ) or higher M-CTC ratio (29 weeks vs. undefined, $\mathrm{HR}=5.085$, 95\% CI: 2.047-12.63, log-rank test $\mathrm{P}=0.0001)$ had decreased RFS compared with other patients.

\section{Dynamic changes in EMT related CTCs total count and subtype count during treatment}

Furthermore, we examined dynamic changes of CTCs total count or M-CTC count before surgery, pre-chemotherapy and after 2 courses of adjuvant therapy. The blood samples of 18 patients who received gemcitabine-based adjuvant therapy were collected, and we found significant difference in CTCs total count between the time points of pre-surgery and post-chemo \{median: $4[1-6]$ vs. $2[1-3] / \mathrm{mL}, \mathrm{P}=0.0230\}$, but no significant difference in CTCs total count between pre-surgery and Pre-chemo, or between Pre-chemo and post-chemo ( $\mathrm{P}=0.1117, \mathrm{P}=0.9999$, respectively) (Figure $3 A$ ).

For M-CTC count, there was no difference between three groups (all $\mathrm{P}=0.9999)$ (Figure 3B). Kaplan-Meier survival analysis showed that M-CTC after 2 courses of chemotherapy was significantly associated with the shorter RFS (49.5 weeks vs. undefined, HR $=7.484$, 95\% CI: $1.462-$ 
Table 1 Correlation between CTCs enumeration or M-CTC percentage with clinicopathological features in PDAC patients

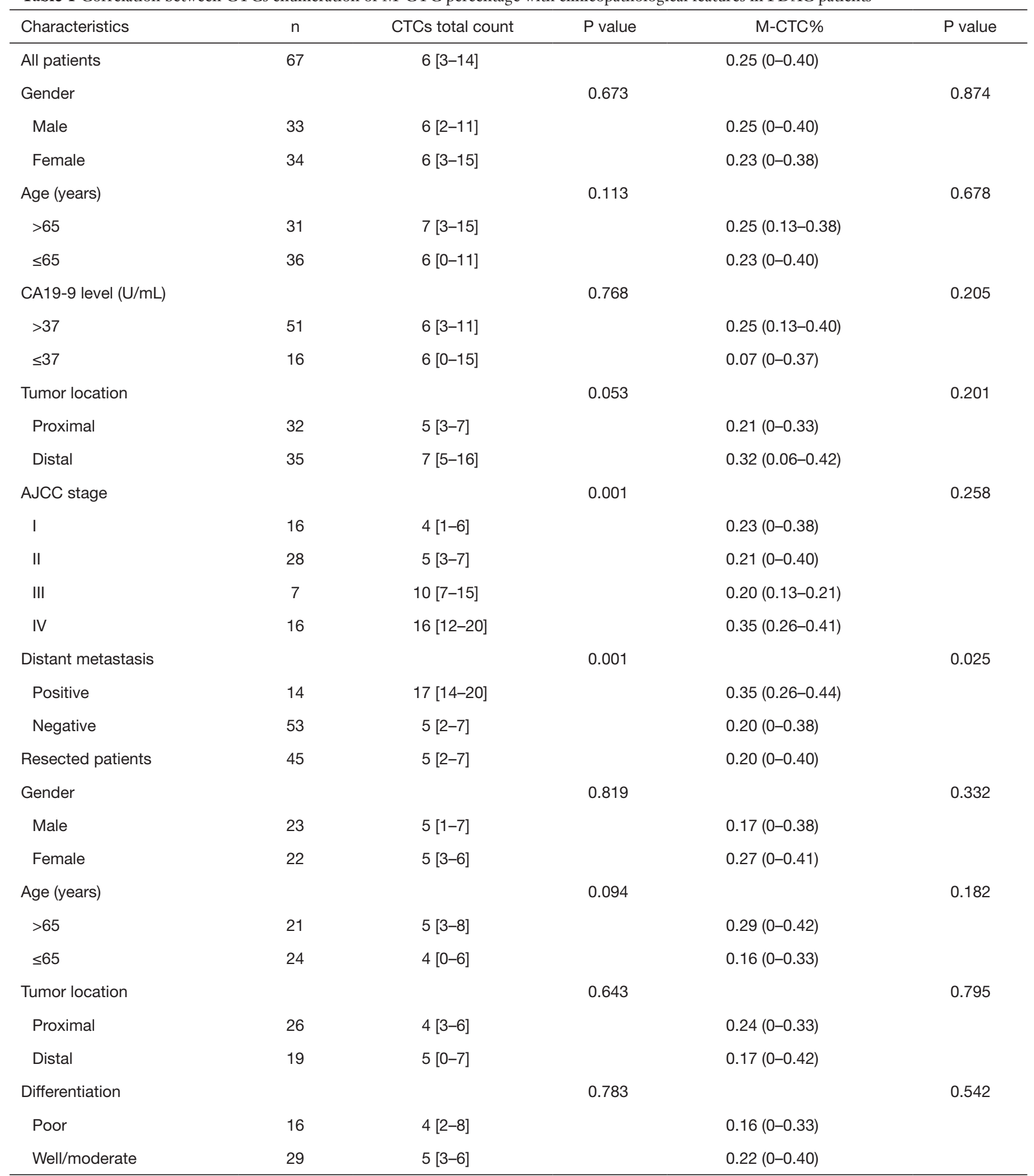

Table 1 (continued) 
Table 1 (continued)

\begin{tabular}{|c|c|c|c|c|c|}
\hline Characteristics & $\mathrm{n}$ & CTCs total count & $P$ value & М-СТС\% & $P$ value \\
\hline$>40$ & 16 & $6[4-8]$ & & $0.33(0-0.42)$ & \\
\hline$\leq 40$ & 29 & $4[2-6]$ & & $0.20(0-0.33)$ & \\
\hline Lymph nodes status & & & 0.783 & & 0.542 \\
\hline Negative & 29 & $5[3-6]$ & & $0.22(0-0.40)$ & \\
\hline Perineural invasion & & & 0.566 & & 0.251 \\
\hline Positive & 42 & $5[2-6]$ & & $0.20(0-0.38)$ & \\
\hline Negative & 3 & $6[3-5]$ & & $0.50(0.25-0.52)$ & \\
\hline Negative & 29 & $4[0-6]$ & & $0.17(0-0.40)$ & \\
\hline
\end{tabular}

CTCs, circulating tumor cells; CA19-9, carbohydrate antigen 19-9; AJCC, American Joint Committee on Cancer.

Table 2 Univariate and multivariate analyses of prognostic factors for RFS in PDAC patients

\begin{tabular}{|c|c|c|c|c|c|c|}
\hline Characteristics & \multicolumn{3}{|c|}{ Univariate analysis } & \multicolumn{3}{|c|}{ Multivariate analysis } \\
\hline Gender (male vs. female) & 1.009 & $0.425-2.394$ & 0.984 & & & \\
\hline Age (>65 vs. $\leq 65$ years) & 1.665 & $0.687-4.033$ & 0.259 & & & \\
\hline CA19-9 level (>37 vs. $\leq 37 \mathrm{U} / \mathrm{mL})$ & 1.410 & $0.473-4.206$ & 0.538 & & & \\
\hline Differentiation (poor vs. well/moderate) & 1.666 & $0.676-4.108$ & 0.267 & & & \\
\hline Tumor size (>40 vs. $\leq 40 \mathrm{~mm}$ ) & 1.986 & $0.840-4.693$ & 0.118 & 1.934 & $0.768-4.870$ & 0.162 \\
\hline Lymph nodes status (positive vs. negative) & 1.666 & $0.676-4.108$ & 0.267 & & & \\
\hline AJCC stage (IIB-III vs. I-IIA) & 2.673 & $1.078-6.631$ & 0.034 & 3.749 & $1.185-11.862$ & 0.025 \\
\hline CTCs total count (>3 vs. $\leq 3 / \mathrm{mL}$ ) & 2.047 & $0.788-5.314$ & 0.141 & 0.503 & $0.141-1.801$ & 0.291 \\
\hline M-CTC percentage (>23.5\% vs. $\leq 23.5 \%$ ) & 5.997 & $2.151-16.725$ & 0.001 & 9.745 & $2.486-38.193$ & 0.001 \\
\hline
\end{tabular}

CTCs, circulating tumor cells; M-CTC, CTCs with mesenchymal phenotype; CA19-9, carbohydrate antigen 19-9; AJCC, American Joint Committee on Cancer.

38.31, log-rank test $\mathrm{P}=0.0288$ ). These data suggested a potential association between M-CTC and chemoresistance, and patients with higher numbers of M-CTC had inferior RFS after surgical resection (Figure 3C).
bENT-1 expression in CTCs and tumor tissues and the correlation with clinicopathological parameters

Peripheral blood samples were collected from 45 patients 
A

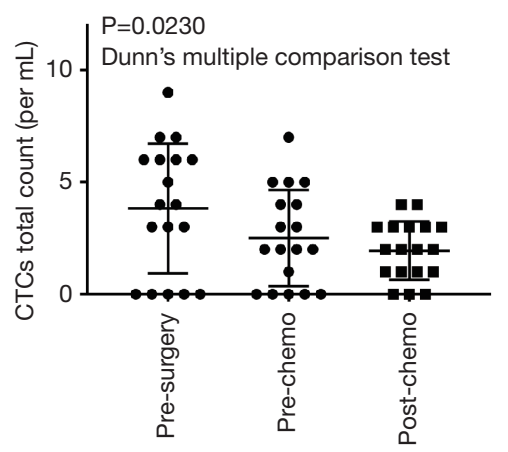

B

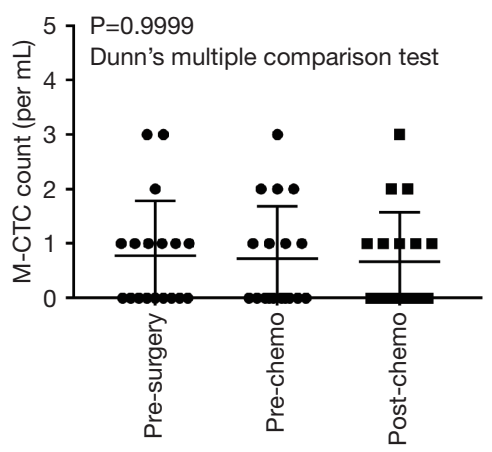

C

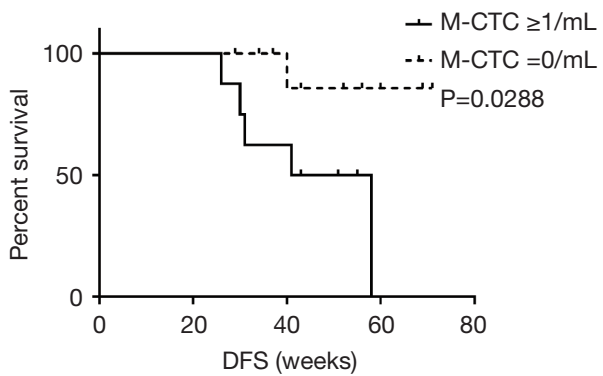

Figure 3 Dynamic changes in CTCs total count and subtype count during treatment. (A) Significant difference in CTCs total count between the time points of pre-surgery and post-chemo \{median: 4 [1-6] vs. 2[1-3]/mL, P=0.0230\}. (B) No significant difference in M-CTC count during chemotherapy (all $\mathrm{P}=0.9999$ ). (C) Kaplan-Meier survival curves showing different RFS in resected PDAC patients with M-CTC $\geq 1 / \mathrm{mL}$ or not at the time of post-chemo (49.5 weeks $v$ s. undefined, HR =7.484, 95\% CI: $1.462-38.31, \log$-rank test $\mathrm{P}=0.0288$ ).

who underwent curative resection, and CTCs were detected in 39/45 (87\%) samples. hENT-1 staining of CTCs showed a median staining score of 4.0 in all samples, 17 patients had a relatively low expression level $(<4.0)$ while 22 had a high expression of hENT-1 ( $\geq 4.0)$. Then we analyzed the correlation of hENT-1 expression with clinicopathological parameters of patients, found that hENT-1 expression in CTCs was only associated with microvascular invasion $(\mathrm{P}=0.008)$ (Table 3). Particularly, the median of M-CTC ratio was $0.33(0.11-0.40)$ in patients with lower hENT-1 expression ( $\mathrm{n}=17)$, and was $0.24(0-0.38)$ in higher hENT-1 expression cohort $(\mathrm{n}=22)$, although there was no significantly difference $(\mathrm{P}=0.510)$.

Among all patients enrolled, 39 patients had a matched tumor tissue to compare hENT-1 staining scores in CTCs and tumor tissues. Statistical analysis showed no correlation in hENT-1 expression status between CTCs and tumor tissues (Figure 4A).

\section{bENT-1 expression in CTCs and tumor tissues for prognostic predication}

Finally, we analyzed prognostic significance of hENT-1 expression in CTCs and tumor tissues. In 39 patients with CTCs hENT-1 score available, univariate analysis of RFS revealed that patients with higher TNM stage $(\mathrm{P}=0.040)$, positive microvascular invasion $(\mathrm{P}=0.022)$ and lower hENT1 expression in CTCs $(\mathrm{P}=0.041)$ had decreased RFS. Multivariate analysis showed that only hENT-1 expression level in CTCs was the independent prognostic factor in resected patients undergoing adjuvant gemcitabine-based chemotherapy $(\mathrm{P}=0.016)$ (Table 4). Log-rank test showed that patients with low expression level in CTCs had decreased RFS (32 weeks vs. undefined, $\mathrm{HR}=2.526,95 \%$ CI: $1.02-6.252, \log$-rank test $\mathrm{P}=0.0337$ ) (Figure $4 B$ ).

\section{Discussion}

As CTCs play a key role in tumor progression, a better understanding of CTCs has great promise to develop novel diagnostic or prognostic biomarkers for PDAC $(32,33)$. In an attempt to establish an efficient and reliable blood test for the diagnosis and survival estimation of PDAC patients, we used CD45 negative enrichment method coupled with 4-channel immunofluorescence staining for phenotypic profiling of CTCs based on EMT or hENT-1. We found that CTCs had heterogeneous phenotypic alterations, and M-CTC rather than CTCs total count had better clinical relevance in either making diagnosis or predicting prognosis. Furthermore, lower hENT-1 expression in CTCs was significantly associated with decreased RFS in resected PDAC patients undergoing adjuvant gemcitabinebased chemotherapy.

Previous studies have suggested that EMT contributes to the dissemination of cancer cells at pre-cancerous stage and is crucial for the invasion and metastasis of PDAC (21-23,34). However, the role of CTCs with EMT features in PDAC development and metastasis remains unclear. In this study, we found that EMT related CTCs could be isolated in the majority of PDAC patients (54/67, 
Table 3 Correlation between hENT-1 expression in CTCs with clinicopathological features in PDAC patients

\begin{tabular}{|c|c|c|c|}
\hline \multirow{2}{*}{ Characteristics } & \multicolumn{2}{|c|}{ CTCs $(n=39)$} & \multirow{2}{*}{$P$ value } \\
\hline & Low & High & \\
\hline Gender & & & 0.855 \\
\hline Male & 8 & 11 & \\
\hline Female & 9 & 11 & \\
\hline Age (years) & & & 0.267 \\
\hline$>65$ & 10 & 9 & \\
\hline$\leq 65$ & 7 & 13 & \\
\hline Tumor location & & & 0.987 \\
\hline Proximal & 10 & 13 & \\
\hline Distal & 7 & 9 & \\
\hline CA19-9 level (U/mL) & & & 0.731 \\
\hline$>37$ & 14 & 19 & \\
\hline$\leq 37$ & 3 & 3 & \\
\hline Differentiation & & & 0.102 \\
\hline Poor & 9 & 6 & \\
\hline Well/moderate & 8 & 16 & \\
\hline Tumor size (mm) & & & 0.759 \\
\hline$>40$ & 7 & 8 & \\
\hline$\leq 40$ & 10 & 14 & \\
\hline Lymph nodes status & & & 0.102 \\
\hline Positive & 9 & 6 & \\
\hline Negative & 8 & 16 & \\
\hline AJCC stage & & & 0.855 \\
\hline IIB-III & 8 & 11 & \\
\hline I-IIA & 9 & 11 & \\
\hline Perineural invasion & & & 0.709 \\
\hline Positive & 16 & 20 & \\
\hline Negative & 1 & 2 & \\
\hline Microvascular invasion & & & 0.008 \\
\hline Positive & 11 & 5 & \\
\hline Negative & 6 & 17 & \\
\hline
\end{tabular}

CTCs, circulating tumor cells; hENT-1, human equilibrative nucleoside transporter-1; CA19-9, carbohydrate antigen 19-9; AJCC, American Joint Committee on Cancer.
$81 \%$ ), indicating that CTCs may be released even in the early stage of PDAC and could be used as a biomarker of early detection. Moreover, the so called circulating epithelial cells (CECs) could be found in the benign and premalignant pancreatic diseases due to inflammatory response (31,35). As a result, malignant CECs isolated via epithelial markers-dependent CTCs detection systems might have limited ability in differentiating a variety of suspicious malignant lesions with PDAC in clinical practice, while CTCs with mesenchymal properties (M-CTC) have a good performance. In addition, both the total count of EMT related CTCs and M-CTC ratio had the ability in differentiating local/regional disease with metastatic disease, implying that EMT related CTCs were significantly correlated with metastatic burden in PDAC patients.

Furthermore, multivariate analysis confirmed that M-CTC percentage was an independent prognostic indicator of RFS in resected PDAC patients. Collectively, our results support the key role of EMT in metastatic potential, and indicate that M-CTC might be better than other sub-populations to predict the risk of disease progression $(36,37)$. Some groups insisted that epithelial-type CTCs with a restricted mesenchymal transition had the strongest metastases formation ability compared with E-CTC or M-CTC, and M-CTC was the subline more resistant to chemotherapy based on animal models (38-40). These results seem to contradict our findings, however, PDAC patients enrolled in our study all received radical surgery followed by gemcitabine-based adjuvant therapy. Patients with a higher M-CTC ratio were more likely to be resistant to current chemotherapy and had a relatively inferior RFS, suggesting that M-CTC analysis might have more clinical impact.

Since peripheral blood samples could be conveniently and frequently collected, the identification of CTCs during treatment process may provide additional information on the responses to current chemotherapy $(17,18,26,27)$. Indeed, post-operative monitoring of CTCs changes could provide an earlier predictor of distant recurrence in hepatocellular carcinoma compared to conventional imaging strategies, because the potential micrometastases may lead to the release of CTCs into the circulation (36). Therefore, in this study we monitored dynamic changes of CTCs in 18 PDAC patients at the time points of pre-surgery, prechemotherapy and 2 courses after chemotherapy. We found that CTCs total count showed significant decrease, while 
A

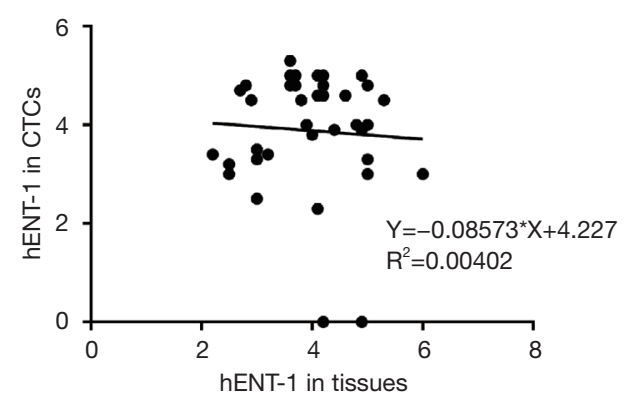

B

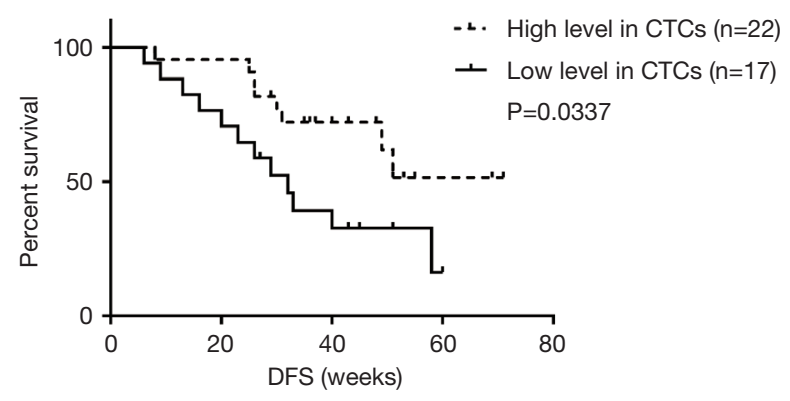

Figure 4 Evaluation of CTCs as prognostic markers in resected PDAC patients undergoing adjuvant gemcitabine-based chemotherapy. (A) Scatter plots for hENT-1 staining score in CTCs and matched tumor tissues. Regression lines (R2=0.00402, $\mathrm{P}=0.7013)$ showed no significant correlation between hENT-1 expression in CTCs and matched tumor tissues. (B) hENT-1 expression level in CTCs as prognostic marker in resected PDAC patients undergoing adjuvant gemcitabine-based chemotherapy. Kaplan-Meier survival curves showing different RFS in PDAC patients with low and high hENT-1 expression level (32 weeks vs. undefined, HR =2.526, 95\% CI: 1.02-6.252, $\log$ rank test $\mathrm{P}=0.0337$ ).

Table 4 Prognostic factors of resected PDAC patients undergoing adjuvant gemcitabine-based chemotherapy

\begin{tabular}{|c|c|c|c|c|c|c|}
\hline Characteristics & \multicolumn{3}{|c|}{ Univariate analysis } & \multicolumn{3}{|c|}{ Multivariate analysis } \\
\hline Gender (male vs. female) & 1.160 & $0.469-2.868$ & 0.748 & & & \\
\hline Age (>65 vs. $\leq 65$ years) & 1.403 & $0.568-3.467$ & 0.463 & & & \\
\hline Tumor location (proximal vs. distal) & 1.340 & $0.556-3.228$ & 0.515 & & & \\
\hline Differentiation (poor vs. well/moderate) & 1.264 & $0.495-3.224$ & 0.624 & & & \\
\hline Tumor size (>40 vs. $\leq 40 \mathrm{~mm}$ ) & 2.020 & $0.837-4.875$ & 0.118 & 1.491 & $0.586-3.790$ & 0.402 \\
\hline Lymph nodes status (positive vs. negative) & 1.264 & $0.495-3.224$ & 0.624 & & & \\
\hline AJCC stage (IIB-III vs. I-IIA) & 2.626 & $1.044-6.603$ & 0.040 & 2.590 & $0.881-7.616$ & 0.084 \\
\hline hENT-1 in tissues (low vs. high) & 2.095 & $0.864-5.079$ & 0.102 & 2.509 & $0.876-7.184$ & 0.087 \\
\hline hENT-1 in CTCs (low vs. high) & 2.549 & $1.038-6.261$ & 0.041 & 3.900 & $1.285-11.834$ & 0.016 \\
\hline
\end{tabular}

CTCs, circulating tumor cells; hENT-1, human equilibrative nucleoside transporter-1; CA19-9, carbohydrate antigen 19-9; AJCC, American Joint Committee on Cancer.

patients with M-CTC after 2 courses of chemotherapy had the shorter RFS, consistent with previous reports that CTCs with mesenchymal phenotypes were associated with chemotherapy resistance and poor prognosis $(36,37,39,41)$.

A previous report suggested that overt micrometastases may have existed at initial diagnosis (13). Michor et al. showed that the risk of micrometastases formation was approximately $28 \%, 73 \%$ and $94 \%$, with the tumor diameters of 1,2 and $3 \mathrm{~cm}$ at the time of diagnosis, respectively (42). Furthermore, the development of chemoresistance to gemcitabine severely limits the effectiveness of current adjuvant therapy to eradicate 
micrometastases, leading to such a high rate of recurrence (4). hENT-1 is a nucleoside transporter protein that plays an important role in transporting cytotoxic chemotherapies such as gemcitabine into tumor cells $(7,8)$. Recently, hENT1 has been reported as a promising predictive biomarker of the treatment outcome of chemotherapy in various cancers (43-45), including PDAC $(9,10,28,29,46)$. However, there is still no consensus on whether hENT-1 expression in resected tumor specimens correlates to the chemosensitivity of the occult micrometastases (13). Since CTCs are proposed as the indicator of residual micrometastases $(24,25)$, whether hENT-1 expression in CTCs could serve as an alternative biomarker to select patients who can truly benefit from gemcitabine treatment to prolong RFS is of great interest.

In this study, our results showed that hENT-1 expression in CTCs may be a promising biomarker in identifying resected PDAC patients with gemcitabine-based adjuvant chemotherapy at higher risk for tumor recurrence. Patients who had lower circulating hENT-1 level had decreased RFS. Furthermore, multivariate analyses confirmed hENT-1 expression in CTCs as the independent prognostic factor. These findings support the possibility that CTCs may play a crucial role in distant metastasis and the elimination of CTCs with high hENT-1 expression by gemcitabine-based therapies may help control micrometastases and improve patient outcome. While previous studies mainly focused on PD-L1 in CTCs of malignant solid tumors $(47,48)$, our study was the first to investigate hENT-1 expression in CTCs and its potential clinical significance in resected patients. Since there are still no effective approaches for risk stratification and neoadjuvant therapies selection among resectable PDAC patients, these results suggest a potential method for pre-operative stratification of patients at high risk of metastasis who may profit from aggressive systemic therapies other than upfront resection, as patients with a higher M-CTC ratio may be more likely to develop early recurrence and are inadvisable to receive current gemcitabine-based regimens due to potential low hENT-1 expression in CTCs.

Interestingly, the present study showed that hENT-1 expression in CTCs showed remarkable discrepancies with its expression in the matched tumor tissues. In our study, the differential expression levels of hENT-1 between them might be related to EMT. It is noted that EMT may suppress cancer cell proliferation and downregulate drug transporter ENT-1 in pancreatic cancer (49). With EMT program, CTCs disseminated from the primary tumor may have distinct biological characteristics (50). Another possible explanation may be the tumor heterogeneity. Previous studies on the discordance in biomarker expression between primary tumor and CTCs suggested the existence of heterogeneous tumor sub-clones that are often not represented in pathological examination $(51,52)$. This could partly explain why hENT-1 expression in CTCs has a better performance in predicting survival outcome than that in tissues in our study. CTCs exhibit the properties of not only the primary tumor but also the metastatic lesions, and could provide more information on therapeutic responses $(16,17,26,27)$.

Our study has some potential limitations. A major limitation is limited sample size in a single institution cohort. Furthermore, this study focused on the analysis of the blood obtained from patients at early stage, and prognostic significance of CTCs in advanced or metastatic PDAC patients should be further analyzed.

\section{Conclusions}

In conclusion, this study suggested that the evaluation of heterogeneous phenotypic profiling of CTCs is instrumental in monitoring the progression and metastasis of PDAC, and CTCs could be used as potential diagnosis and prognosis biomarkers for PDAC patients.

\section{Acknowledgments}

The authors also thank Yan Zhuang, Zhengfei Zhou, Hongqiao Gao, Xuehai Xie, Yiran Chen, Shouge Zang and Jing Zhu from our department for their collection and preparation of the samples.

Funding: This work was supported in part by grants 81672353,81871954 and 82171722 from the National Natural Science Foundation of China (to Y Yang); grant 7212111 from the Beijing Municipal Natural Science Foundation (to Y Yang); grant 2019CR18 from the Youth Clinical Research Project of Peking University First Hospital (to Y Ma); grant 2021SF26 from the Youth Clinical Research Project of Peking University First Hospital (to X Zhao) and grant SS2015AA020405 from the National High Technology Research and Development Program of China (to T Liang).

\section{Footnote}

Reporting Checklist: The authors have completed the 
REMARK reporting checklist. Available at https://hbsn. amegroups.com/article/view/10.21037/hbsn-20-383/rc

Data Sharing Statement: Available at https://hbsn. amegroups.com/article/view/10.21037/hbsn-20-383/dss

Conflicts of Interest: All authors have completed the ICMJE uniform disclosure form (available at https://hbsn. amegroups.com/article/view/10.21037/hbsn-20-383/coif). Dr. YMY serves as an unpaid editorial board members of Hepatobiliary Surgery and Nutrition. The other authors have no conflicts of interest to declare.

Ethical Statement: The authors are accountable for all aspects of the work in ensuring that questions related to the accuracy or integrity of any part of the work are appropriately investigated and resolved. The study was conducted in accordance with the Declaration of Helsinki (as revised in 2013). The study was approved by the Institutional Review Board at the Peking University First Hospital (No. 2018 keyan-15) and informed consent was taken from all individual participants.

Open Access Statement: This is an Open Access article distributed in accordance with the Creative Commons Attribution-NonCommercial-NoDerivs 4.0 International License (CC BY-NC-ND 4.0), which permits the noncommercial replication and distribution of the article with the strict proviso that no changes or edits are made and the original work is properly cited (including links to both the formal publication through the relevant DOI and the license). See: https://creativecommons.org/licenses/by-nc-nd/4.0/.

\section{References}

1. Rahib L, Smith BD, Aizenberg R, et al. Projecting cancer incidence and deaths to 2030: the unexpected burden of thyroid, liver, and pancreas cancers in the United States. Cancer Res 2014;74:2913-21.

2. Groot VP, Rezaee N, Wu W, et al. Patterns, Timing, and Predictors of Recurrence Following Pancreatectomy for Pancreatic Ductal Adenocarcinoma. Ann Surg 2018;267:936-45.

3. Jones RP, Psarelli EE, Jackson R, et al. Patterns of Recurrence After Resection of Pancreatic Ductal Adenocarcinoma: A Secondary Analysis of the ESPAC-4 Randomized Adjuvant Chemotherapy Trial. JAMA Surg 2019;154:1038-48.
4. Groot VP, Gemenetzis G, Blair AB, et al. Defining and Predicting Early Recurrence in 957 Patients With Resected Pancreatic Ductal Adenocarcinoma. Ann Surg 2019;269:1154-62.

5. Kleeff J, Korc M, Apte M, et al. Pancreatic cancer. Nat Rev Dis Primers. 2016;2:16022.

6. Tempero MA, Malafa MP, Chiorean EG, et al. Pancreatic Adenocarcinoma, Version 1.2019. J Natl Compr Canc Netw 2019;17:202-10.

7. Andersson R, Aho U, Nilsson BI, et al. Gemcitabine chemoresistance in pancreatic cancer: molecular mechanisms and potential solutions. Scand J Gastroenterol. 2009. 44(7): 782-6.

8. Yao SY, Ng AM, Cass CE, et al. Nucleobase transport by human equilibrative nucleoside transporter 1 (hENT1). J Biol Chem 2011;286:32552-62.

9. Farrell JJ, Elsaleh H, Garcia M, et al. Human equilibrative nucleoside transporter 1 levels predict response to gemcitabine in patients with pancreatic cancer. Gastroenterology 2009;136:187-95.

10. Nakagawa N, Murakami Y, Uemura K, et al. Combined analysis of intratumoral human equilibrative nucleoside transporter 1 (hENT1) and ribonucleotide reductase regulatory subunit M1 (RRM1) expression is a powerful predictor of survival in patients with pancreatic carcinoma treated with adjuvant gemcitabine-based chemotherapy after operative resection. Surgery 2013;153:565-75.

11. Sinn M, Riess H, Sinn BV, et al. Human equilibrative nucleoside transporter 1 expression analysed by the clone SP 120 rabbit antibody is not predictive in patients with pancreatic cancer treated with adjuvant gemcitabine - Results from the CONKO-001 trial. Eur J Cancer 2015;51:1546-54.

12. Jiraskova L, Ryska A, Duintjer Tebbens EJ, et al. Are ENT1/ENT1, NOTCH3, and miR-21 Reliable Prognostic Biomarkers in Patients with Resected Pancreatic Adenocarcinoma Treated with Adjuvant Gemcitabine Monotherapy?. Cancers (Basel) 2019;11:1621.

13. Tuveson DA, Neoptolemos JP. Understanding metastasis in pancreatic cancer: a call for new clinical approaches. Cell 2012;148:21-3.

14. Daamen LA, Groot VP, Heerkens HD, et al. Systematic review on the role of serum tumor markers in the detection of recurrent pancreatic cancer. HPB (Oxford) 2018;20:297-304.

15. Lee AJ, Simoneau E, Chiang YJ, et al. Is early-stage pancreatic adenocarcinoma truly early: stage migration 
on final pathology with surgery-first versus neoadjuvant therapy sequencing. HPB (Oxford) 2019;21:1203-10.

16. Plaks V, Koopman CD, Werb Z. Cancer. Circulating tumor cells. Science 2013;341:1186-8.

17. Nagrath S, Jack RM, Sahai V, et al. Opportunities and Challenges for Pancreatic Circulating Tumor Cells. Gastroenterology 2016;151:412-26.

18. Buscail E, Maulat C, Muscari F, et al. Liquid Biopsy Approach for Pancreatic Ductal Adenocarcinoma. Cancers (Basel) 2019;11:852.

19. Ankeny JS, Court CM, Hou S, et al. Circulating tumour cells as a biomarker for diagnosis and staging in pancreatic cancer. Br J Cancer 2016;114:1367-75.

20. Wang Y, Yu X, Hartmann D, et al. Circulating tumor cells in peripheral blood of pancreatic cancer patients and their prognostic role: a systematic review and metaanalysis. HPB (Oxford) 2020;22:660-9. doi:10.1016/ j.hpb.2019.11.003.

21. Rhim AD, Mirek ET, Aiello NM, et al. EMT and dissemination precede pancreatic tumor formation. Cell 2012;148:349-61.

22. Aiello NM, Maddipati R, Norgard RJ, et al. EMT Subtype Influences Epithelial Plasticity and Mode of Cell Migration. Dev Cell 2018;45:681-695.e4.

23. Pastushenko I, Brisebarre A, Sifrim A, et al. Identification of the tumour transition states occurring during EMT. Nature 2018;556:463-8.

24. Joosse SA, Gorges TM, Pantel K. Biology, detection, and clinical implications of circulating tumor cells. EMBO Mol Med 2015;7:1-11.

25. Pantel K, Alix-Panabières C. Liquid biopsy and minimal residual disease - latest advances and implications for cure. Nat Rev Clin Oncol 2019;16:409-424.

26. Khoo BL, Grenci G, Jing T, et al. Liquid biopsy and therapeutic response: Circulating tumor cell cultures for evaluation of anticancer treatment. Sci Adv 2016;2:e1600274.

27. Bardelli A, Pantel K. Liquid Biopsies, What We Do Not Know (Yet). Cancer Cell 2017;31:172-9.

28. Morinaga S, Nakamura Y, Watanabe T, et al. Immunohistochemical analysis of human equilibrative nucleoside transporter-1 (hENT1) predicts survival in resected pancreatic cancer patients treated with adjuvant gemcitabine monotherapy. Ann Surg Oncol 2012;19 Suppl 3:S558-64.

29. Aoyama T, Kazama K, Miyagi Y, et al. Predictive role of human equilibrative nucleoside transporter 1 in patients with pancreatic cancer treated by curative resection and gemcitabine-only adjuvant chemotherapy. Oncol Lett 2017;14:599-606.

30. Waddell N, Pajic M, Patch AM, et al. Whole genomes redefine the mutational landscape of pancreatic cancer. Nature 2015;518:495-501.

31. Cauley CE, Pitman MB, Zhou J, et al. Circulating Epithelial Cells in Patients with Pancreatic Lesions: Clinical and Pathologic Findings. J Am Coll Surg 2015;221:699-707.

32. Cortés-Hernández LE, Eslami-S Z, Alix-Panabières C. Circulating tumor cell as the functional aspect of liquid biopsy to understand the metastatic cascade in solid cancer. Mol Aspects Med 2020;72:100816.

33. Habib Joseph R, Jun Y. Circulating tumor cells in pancreatic cancer: a review. Journal of Pancreatology 2019;2:54-9.

34. Rodriguez-Aznar E, Wiesmüller L, Sainz B Jr, et al. EMT and Stemness-Key Players in Pancreatic Cancer Stem Cells. Cancers (Basel) 2019;11:1136.

35. Rosenbaum MW, Cauley CE, Kulemann B, et al. Cytologic characteristics of circulating epithelioid cells in pancreatic disease. Cancer Cytopathol 2017;125:332-340.

36. Qi LN, Xiang BD, Wu FX, et al. Circulating Tumor Cells Undergoing EMT Provide a Metric for Diagnosis and Prognosis of Patients with Hepatocellular Carcinoma. Cancer Res 2018;78:4731-44.

37. Zhao XH, Wang ZR, Chen CL, et al. Molecular detection of epithelial-mesenchymal transition markers in circulating tumor cells from pancreatic cancer patients: Potential role in clinical practice. World J Gastroenterol 2019;25:138-50.

38. Chen Y, LeBleu VS, Carstens JL, et al. Dual reporter genetic mouse models of pancreatic cancer identify an epithelial-to-mesenchymal transition-independent metastasis program. EMBO Mol Med 2018;10:e9085.

39. Liu X, Li J, Cadilha BL, et al. Epithelial-type systemic breast carcinoma cells with a restricted mesenchymal transition are a major source of metastasis. Sci Adv 2019;5:eaav4275.

40. Williams ED, Gao D, Redfern A, et al. Controversies around epithelial-mesenchymal plasticity in cancer metastasis. Nat Rev Cancer 2019;19:716-32.

41. Wei T, Zhang X, Zhang Q, et al. Vimentin-positive circulating tumor cells as a biomarker for diagnosis and treatment monitoring in patients with pancreatic cancer. Cancer Lett 2019;452:237-43.

42. Haeno H, Gonen M, Davis MB, et al. Computational modeling of pancreatic cancer reveals kinetics of metastasis suggesting optimum treatment strategies. Cell 
2012;148:362-75.

43. Wu F, Zhang J, Hu N, et al. Effect of hENT1 polymorphism G-706C on clinical outcomes of gemcitabine-containing chemotherapy for Chinese non-small-cell lung cancer patients. Cancer Epidemiol 2014;38:728-32.

44. Brandi G, Deserti M, Vasuri F, et al. Membrane Localization of Human Equilibrative Nucleoside Transporter 1 in Tumor Cells May Predict Response to Adjuvant Gemcitabine in Resected Cholangiocarcinoma Patients. Oncologist 2016;21:600-607.

45. Vincenzi B, Stacchiotti S, Collini P, et al. Human equilibrative nucleoside transporter 1 gene expression is associated with gemcitabine efficacy in advanced leiomyosarcoma and angiosarcoma. Br J Cancer 2017;117:340-346.

46. Greenhalf W, Ghaneh P, Neoptolemos JP, et al. Pancreatic cancer hENT1 expression and survival from gemcitabine in patients from the ESPAC-3 trial. J Natl Cancer Inst 2014;106:djt347.

47. Ilié M, Szafer-Glusman E, Hofman V, et al. Detection of

Cite this article as: Zhao X, Ma Y, Dong X, Zhang Z, Tian X, Zhao X, Yang Y. Molecular characterization of circulating tumor cells in pancreatic ductal adenocarcinoma: potential diagnostic and prognostic significance in clinical practice. HepatoBiliary Surg Nutr 2021;10(6):796-810. doi: 10.21037/ hbsn-20-383
PD-L1 in circulating tumor cells and white blood cells from patients with advanced non-small-cell lung cancer. Ann Oncol 2018;29:193-9.

48. Yue C, Jiang Y, Li P, et al. Dynamic change of PD-L1 expression on circulating tumor cells in advanced solid tumor patients undergoing PD-1 blockade therapy. Oncoimmunology 2018;7:e1438111.

49. Zheng X, Carstens JL, Kim J, et al. Epithelial-tomesenchymal transition is dispensable for metastasis but induces chemoresistance in pancreatic cancer. Nature 2015;527:525-30.

50. Lambert AW, Pattabiraman DR, Weinberg RA. Emerging Biological Principles of Metastasis. Cell 2017;168:670-91.

51. Markou A, Farkona S, Schiza C, et al. PIK3CA mutational status in circulating tumor cells can change during disease recurrence or progression in patients with breast cancer. Clin Cancer Res 2014;20:5823-34.

52. Koh Y, Yagi S, Akamatsu H, et al. Heterogeneous Expression of Programmed Death Receptor-ligand 1 on Circulating Tumor Cells in Patients With Lung Cancer. Clin Lung Cancer 2019;20:270-7.e1. 


\section{hENT-1 antibody assessment}

\section{Cell lines}

Human pancreatic tumor cell lines PANC-1, AsPC-1, BxPC-3, CFPAC-1 were obtained from the ATCC (Manassas, VA, USA) and cultured according to the guidelines. All cell lines were authenticated using a short tandem repeat (STR) DNA test.

\section{Western blot analysis}

The cells were lysed in RIPA buffer $[25 \mathrm{mM}$ Tris- $\mathrm{HCl}(\mathrm{pH}$ 7.5), $150 \mathrm{mM} \mathrm{NaCl}, 1 \% \mathrm{NP}-40,1 \%$ sodium deoxycholate, and $0.1 \%$ SDS] supplemented with protease inhibitor cocktail (Roche Applied Science, Germany). The protein concentration was measured using the BCA Protein Assay (Thermo Fisher Scientific, Waltham, MA, USA). Total cell lysates $(30 \mu \mathrm{g})$ were separated on $10 \%$ SDS-PAGE and transferred to a polyvinylidene difluoride membrane (Millipore). The membranes were incubated with the following antibodies: anti-ENT-1 (Abcam), anti- $\beta$-actin (Cell Signaling). Following washing, the membranes were developed with anti-mouse or rabbit horseradish peroxidase conjugated secondary antibodies (Cell Signaling), and analyzed with ImageQuant LAS4000 system (Fujifilm, Tokyo, Japan).

\section{Quantitative real-time reverse transcriptase-polymerase chain reaction ( $R T-q P C R)$}

Total RNA was extracted with TRIzol reagent (Life Technologies), and complementary DNA was synthesized with the SuperScript ${ }^{\mathrm{TM}}$ III First-Strand Synthesis System (Life Technologies). PCR assay was performed in triplicate using a SYBR Premix Dimer Eraser
(Perfect Real Time) kit (Takara Bio, Shiga, Japan) on a CFX96 Touch $^{\mathrm{TM}}$ Real-Time PCR Detection System (Bio-Rad). RPLP0 gene was used as an endogenous control. The primers were as follows: hENT-1: forward 5 '-GTAGCCTCGGCATTTGCTTG-3', reverse 5'-CCTCTGAAGGCACCTGGTT-3'; RPLP0: forward 5 '-CAGATTGGCTACCCAACTGTT-3', reverse 5'-GGGAAGGTGTAATCCGTCTCC-3'.

\section{Immunofluorescence}

The cells grown on glass coverslips were washed using phosphate-buffered saline (PBS) and fixed in 4\% paraformaldehyde for $20 \mathrm{~min}$ at room temperature. Then, the cells were blocked with $2 \%$ bovine serum albumin (BSA) for $30 \mathrm{~min}$. Next, the cells were incubated with antiENT-1 antibody overnight at $4{ }^{\circ} \mathrm{C}$, washed three times, and incubated with Alexa flour 488-conjugated goat anti-rabbit $\mathrm{IgG}$ for $1 \mathrm{~h}$ at room temperature. Finally, the cells were examined using a fluorescence microscope (Nikon, Japan).

\section{Results}

RT-qPCR and Western blot analysis of hENT-1 expression in four different pancreatic cancer cell lines PANC-1, AsPC-1, BxPC-3, CFPAC-1 showed differential hENT1 expression levels among cell lines, and PANC-1 cells had significantly higher hENT-1 expression level, while other cells had lower level (Figure S1A,S1B). Immunofluorescence identification of two different cell lines was in accordance with the results from Western blotting and RT-qPCR. Therefore, the anti-ENT-1 antibody was suitable for immunofluorescence assay and could be used to assess hENT-1 expression level (Figure S1C). 
A

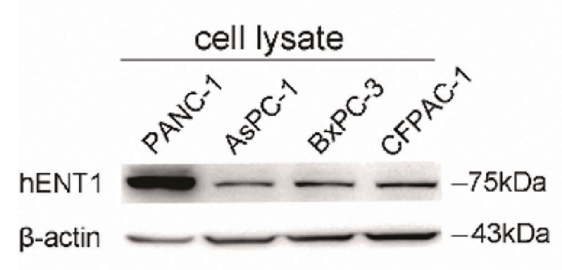

B

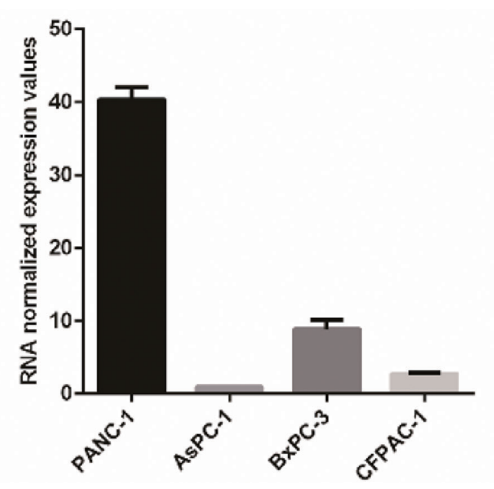

C

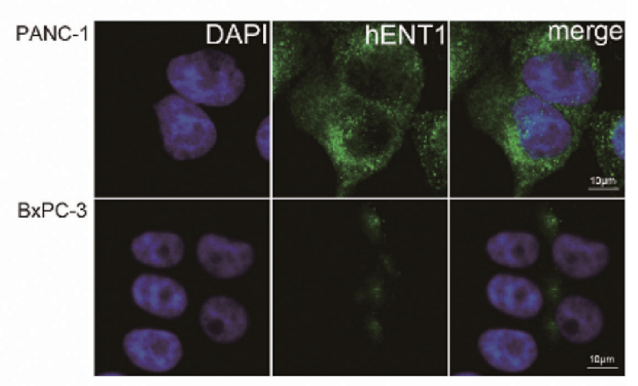

Figure S1 hENT-1 antibody assessment. (A) Western blot analysis of hENT-1 protein expression in four pancreatic cancer cell lines. (B) RT-qPCR of hENT-1 mRNA expression in four pancreatic cancer cell lines. (C) Immunofluorescence identification of two different cell lines PANC-1 and BxPC-3. 